\author{
MITSUBISHI ELECTRIC RESEARCH LABORATORIES \\ http://www.merl.com
}

\title{
Experimental Demonstration of 24-Dimensional Extended Golay Coded Modulation with LDPC
}

\author{
Millar, D.S.; Koike-Akino, T.; Maher, R.; Lavery, D.; Paskov, M.; Kojima, K.; Parsons, K.; \\ Thomsen, B.C.; Savory, S.J.; Bayvel, P.
}

TR2014-010 March 2014

\begin{abstract}
We experimentally demonstrate ultra-long haul transmission of 24-D extended Golay coded modulation with LDPC. Compared with LDPC coded DP-BPSK, an increase of $15 \%$ in reach was shown, with a $3 \mathrm{~dB}$ increase in launch power margin at a transmission distance of more than $16,000 \mathrm{~km}$.

Optical Fiber Communication Conference and Exposition (OFC)
\end{abstract}

This work may not be copied or reproduced in whole or in part for any commercial purpose. Permission to copy in whole or in part without payment of fee is granted for nonprofit educational and research purposes provided that all such whole or partial copies include the following: a notice that such copying is by permission of Mitsubishi Electric Research Laboratories, Inc.; an acknowledgment of the authors and individual contributions to the work; and all applicable portions of the copyright notice. Copying, reproduction, or republishing for any other purpose shall require a license with payment of fee to Mitsubishi Electric Research Laboratories, Inc. All rights reserved.

Copyright (C) Mitsubishi Electric Research Laboratories, Inc., 2014

201 Broadway, Cambridge, Massachusetts 02139 



\title{
Experimental Demonstration of 24-Dimensional Extended Golay Coded Modulation with LDPC
}

\author{
David S. Millar ${ }^{1}$, Toshiaki Koike-Akino' ${ }^{1}$ Robert Maher ${ }^{2}$, Domaniç Lavery ${ }^{2}$, \\ Milen Paskov ${ }^{2}$, Keisuke Kojima ${ }^{1}$, Kieran Parsons ${ }^{1}$, Benn C. Thomsen ${ }^{2}$, \\ Seb J. Savory ${ }^{2}$ and Polina Bayvel ${ }^{2}$ \\ ${ }^{1}$ Mitsubishi Electric Research Laboratories, 201 Broadway, Cambridge, MA 02139, USA: millar@merl.com \\ ${ }^{2}$ Optical Networks Group, University College London (UCL), Torrington Place, London, WC1E 7JE, UK
}

\begin{abstract}
We experimentally demonstrate ultra-long haul transmission of 24-D extended Golay coded modulation with LDPC. Compared with LDPC coded DP-BPSK, an increase of $15 \%$ in reach was shown, with a $3 \mathrm{~dB}$ increase in launch power margin at a transmission distance of more than $16,000 \mathrm{~km}$.
\end{abstract}

OCIS codes: (060.4510) Optical communications, (060.1660) Coherent communications, (060.4080) Modulation.

\section{Introduction}

Modulation for coherent optical systems has become a mature research field in recent years. As ever increasing capacity, noise tolerance and robustness to fiber nonlinearity are required, researchers have investigated modulation and coding techniques as a means of improving performance in these areas. Particularly of interest have been modulation formats which are optimized in four dimensions (4-D) [1-3]. More recently, modulation in 8-D [4,5] and 24-D [6] has been examined over a single-mode optical fiber channel. Increasing the dimensionality of the signal space enables more efficient packing of constellation points and improvements in tolerance to noise and nonlinearity [7,8]. Research has also been undertaken into the performance of high-dimensional and coded modulation formats with soft decision (SD) forward error correction (FEC), such as low density parity check (LDPC) coding [9, 10].

In this paper, we present the first experimental demonstration of 12-bit, 24-D Golay coded hypercube (12b-24DGCHC) modulation (recently proposed in [6]) with LDPC. Transmission performance was characterized over an Erbium doped fiber amplified (EDFA) recirculating loop with a $100.7 \mathrm{~km}$ span of ultra-low loss Corning® SMF-28 ULL ${ }^{\circledR}$ fiber. Compared with LDPC coded dual-polarization binary phase shift keying (DP-BPSK) modulation at the same bit rate and spectral efficiency, we found an increase in reach of $15 \%$ for single channel transmission, and an increase in launch power margin of $3 \mathrm{~dB}$ for a WDM system with $1.05 \mathrm{~b} / \mathrm{s} / \mathrm{Hz}$ net spectral efficiency.

\section{24-Dimensional extended Golay coded modulation with LDPC coding}

24-dimensional modulation using the extended Golay code has been previously shown to offer significant benefits in performance over DP-BPSK at the same spectral efficiency. This is due to the large Hamming distance between extended Golay codewords, and the fact that Hamming distance is proportional to Euclidian distance when a BPSK per dimension modulation is used [6]. Here we experimentally investigate LDPC coded performance of 12b-24DGCHC modulation. In this experiment, we have assumed the use of an outer hard decision (HD) code with a 7\% overhead which can correct the output of the LDPC decoder from a bit error rate (BER) of up to $3.8 \times 10^{-3}$ to a BER of $10^{-15}$ or better. Although this code was not implemented, it enables us to reliably characterize performance at a BER of $10^{-15}$ even though we can only measure to $10^{-5}$ reliably due to experimental limitations. For all modulation formats, we have used an LDPC code with $12.5 \%$ overhead, leading to a total FEC overhead of $20.5 \%$. The LDPC codes were designed according to the procedures defined in [11], and had a girth of 5. Codeword length was 1996 for single-channel experiments and 19800 for the WDM experiments.

Our LDPC encoded data was then interleaved, and used to generate 12b-24D-GCHC signals. We generated 24D signals by mapping 12 information bits to 24 coded bits using the extended Golay code generator matrix. Each extended Golay codeword was then sent in series to a single quadrature in our transmitter. Although coding across quadratures may give improved tolerance to polarization dependent loss (PDL), we were unable to implement this as we only had access to a two lane pulse pattern generator (PPG). In the receiver, an ML detector was used in order to determine the 12 bit-wise log-likelihood ratios (LLRs) for each 12b-24D-GCHC codeword. These LLRs were then 
passed to the de-interleaver, which reordered each interleaver block into its constituent LDPC codewords. The LLRs were then passed to the LDPC decoder, which used 16 iterations of the sum-product algorithm (SPA). Hard decisions were then taken and used to calculate the bit error rate (BER) value of the measurement.

\section{Experimental setup}

The experimental setup is shown in Fig. 1. The encoded data was loaded onto a programmable two channel PPG operating at $28 \mathrm{GBd}$, with a hardware delay of half of the pattern length to de-correlate the in-phase and quadrature driving signals. For WDM measurements, the differential pairs of each PPG lane were used to generate the driving signals for the odd and even channels. An external cavity laser (ECL) with linewidth of $20 \mathrm{kHz}$ was used for the central channel, while in the WDM setup all other carriers were distributed feedback (DFB) sources, spaced at 40 GHz. Modulation was performed with one (single channel) or two (WDM) Mach-Zehnder I/Q modulators. In the WDM case, odd and even channels were modulated independently before interleaving on a $40 \mathrm{GHz}$ grid with a Finisar Waveshaper, and polarization multiplexing.

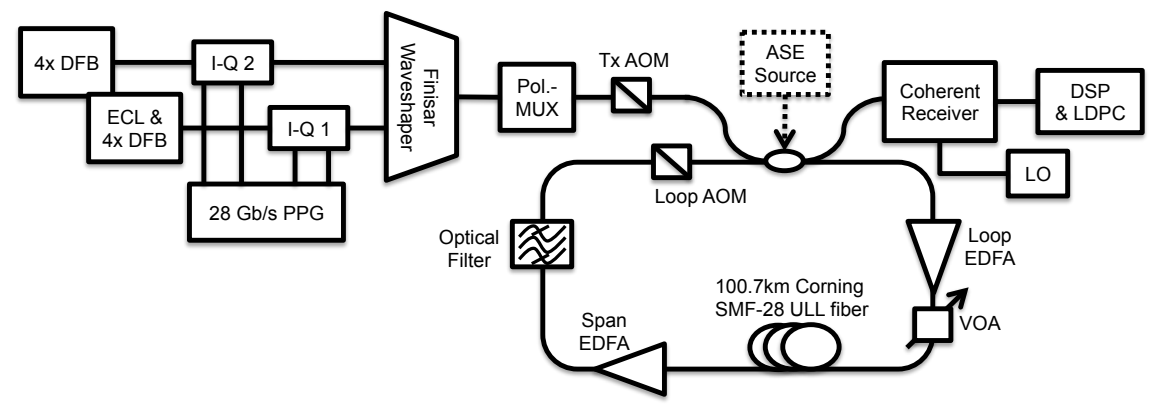

Fig. 1. Experimental setup for transmission experiments. Single channel experiments were performed with only the central channel in operation, and back-to-back experiments were performed with an ASE source in place of the recirculating loop.

Back-to-back performance was characterized by loading the signal with amplified spontaneous emission (ASE) noise of varying power, before detection. Transmission performance was emulated using a single span recirculating loop with a $100.7 \mathrm{~km}$ span of ultra-low loss Corning SMF-28 ULL fiber. The fiber had a span loss of $16 \mathrm{~dB}$ (including splices), chromatic dispersion of $16.3 \mathrm{ps} / \mathrm{nm} / \mathrm{km}$ at $1550 \mathrm{~nm}$ and PMD of less than $0.1 \mathrm{ps} / \mathrm{km}^{1 / 2}$. A loop synchronous polarization scrambler was used to randomize the polarization state of the loop and mitigate the effects of polarization dependent loss or gain (PDL/PDG). The signals were detected with a dual-polarization coherent receiver with overall bandwidth of approximately $20 \mathrm{GHz}$, which used an ECL with linewidth of $100 \mathrm{kHz}$ for the local oscillator. The electrical signals from the receiver front end were then captured using a real-time oscilloscope with $20 \mathrm{GHz}$ bandwidth and a $50 \mathrm{GSa} / \mathrm{s}$ sampling rate, before being processed offline in Matlab.

\section{Results and discussion}

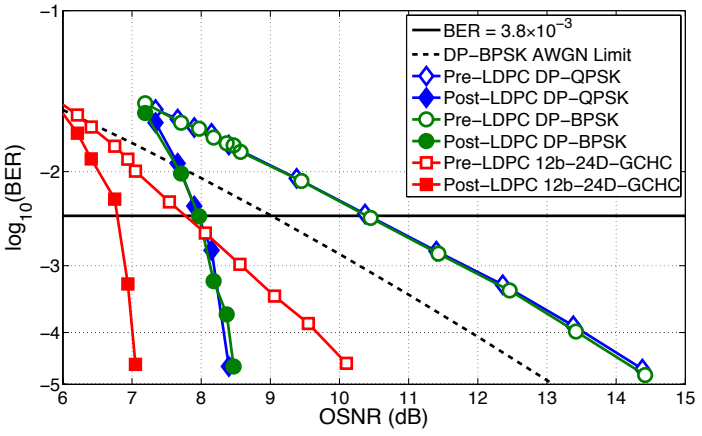

(a)

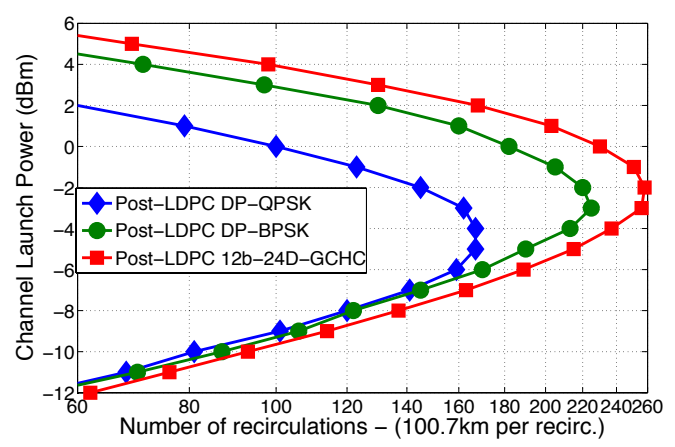

(b)

Fig. 2. Single channel results comparing LDPC coded DP-BPSK and DP-QPSK with LDPC and extended Golay coded QPSK at a net bit rate of $46.6 \mathrm{~Gb} / \mathrm{s}$ (including FEC overhead). Back-to-back results are presented in (a), while maximum reach assuming an outer HD-FEC BER threshold of $3.8 \times 10^{-3}$ is presented in (b).

Firstly, we characterized back-to-back performance of single channel 12b-24D-GCHC and compared it with DPBPSK and DP-QPSK. Each format used the same LDPC code and had the same total bit rate of $56 \mathrm{~Gb} / \mathrm{s}$ corresponding to a net bit rate of $46.5 \mathrm{~Gb} / \mathrm{s}$ after FEC overhead. These results are presented in Fig. 2(a). We note that the uncoded results show the expected behavior: DP-BPSK and DP-QPSK have identical noise tolerance, while 12b-24D-GCHC 
modulation exhibits an improvement in noise tolerance of approximately $2.5 \mathrm{~dB}$ at a BER of $3.8 \times 10^{-3}$. After LDPC decoding, we note that again this gain is reduced to around $1.2 \mathrm{~dB}$. Single channel transmission performance was then characterized, the results of which are shown in Fig. 2(b). We note the expected improvement in the linear region is consistent with our back-to-back characterization, while in the nonlinear region, DP-BPSK and 12b-24D-GCHC show significant gains due to their broader spectra [12]. DP-QPSK has a maximum reach of 167 spans and an optimum launch power of $-4 \mathrm{dBm}$, while DP-BPSK has a maximum reach of 225 spans and an optimum launch power of $-3 \mathrm{dBm}$. 12b-24D-GCHC has an optimum launch power of $-2 \mathrm{dBm}$ and a maximum reach of 258 spans, corresponding to $25,980 \mathrm{~km}$ - an increase in reach of $15 \%$ compared with DP-BPSK.

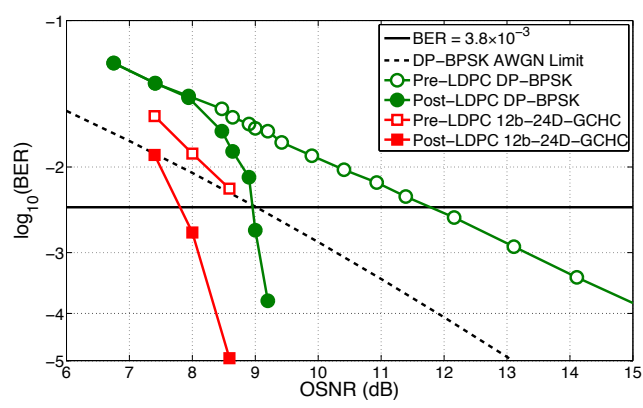

(a)

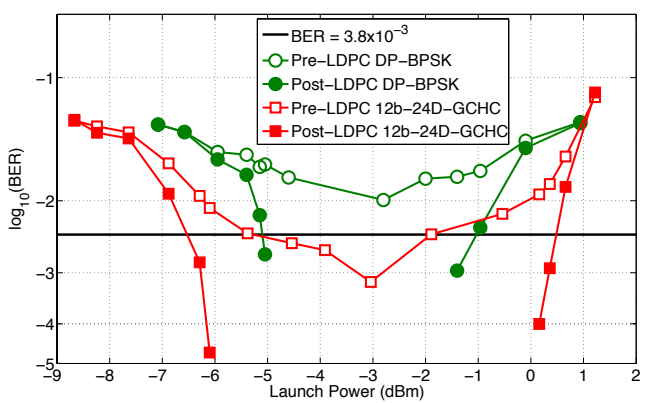

(b)

Fig. 3. WDM results comparing LDPC coded DP-BPSK with LDPC and extended Golay coded QPSK at a net bit rate of 46.6 Gb/s (including FEC overhead) with a $40 \mathrm{GHz}$ channel spacing. Back-to-back results are presented in (a), while BER vs. launch power per channel at 160 recirculations $(16,112 \mathrm{~km})$ is presented in (b).

We then characterized the performance of a 9 channel WDM system on a $40 \mathrm{GHz}$ grid, leading to a net SE of $1.05 \mathrm{~b} / \mathrm{s} / \mathrm{Hz}$. The back-to-back characterization is presented in Fig. 3(a). We note that the implementation penalty has increased to approximately $2.7 \mathrm{~dB}$ due to optical filtering and crosstalk for both DP-BPSK and 12b-24D-GCHC. WDM transmission performance was characterized at a fixed distance of 160 spans $(16,110 \mathrm{~km})$, and the results are presented in Fig. 3(b). We note that the range of launch powers which achieve the target LDPC decoder output BER is increased from $4 \mathrm{~dB}$ to $7 \mathrm{~dB}$ - an improvement of $3 \mathrm{~dB}$.

\section{Conclusions}

We have demonstrated transmission of LDPC coded 24-dimensional extended Golay coded modulation and compared it with LDPC coded DP-BPSK at a net bit rate of $46.5 \mathrm{~Gb} / \mathrm{s}$. OSNR sensitivity was improved by $1.2 \mathrm{~dB}$ at an LDPC decoder output BER of $3.8 \times 10^{-3}$ compared with DP-BPSK for both single channel and WDM experiments. For single channel transmission, maximum reach was increased by $15 \%$ from 22,660 $\mathrm{km}$ to $25,980 \mathrm{~km}$. WDM performance was characterized at a distance of $16,110 \mathrm{~km}$, and launch power margin was found to improve by $3 \mathrm{~dB}$.

\section{Acknowledgements}

The authors would like to thank Dr. Sergejs Makovejs and Corning Inc. for the loan of the fiber, and Yoshikuni Miyata, Wataru Matsumoto, Takashi Sugihara, Kazuyuki Ishida, Tsuyoshi Yoshida and Takashi Mizuochi at Mitsubishi Electric Corp. in Japan for useful discussions.

\section{References}

1. E. Agrell \& M. Karlsson, J. Lightw. Tech., 2722: 5115-5126, 2009.8. P. Poggiolini et al., Opt. Exp., 18, 11360-11371, 2010.

2. M. Karlsson \& E. Agrell, Opt. Exp. 1713: 10814-10819, 2009. $\quad$ 9. D. G. Foursa et al., Proc. ECOC 2013, PD3.E.1.

3. H. Bülow, X. Lu, \& L. Schmalen, Proc. ECOC 2013, Mo.4.D.2. 10. I. B. Djordevic et al., IEEE Photonics Journ., 54, pp. 7901312-

4. T. A. Eriksson et al., Proc. ECOC 2013, Th.2.D.4.

5. T. Koike-Akino et al., Proc. ECOC 2013, Tu.3.C.3.

6. D. S. Millar et al., Proc. SPPCOM 2013, SPM3D.6. 7901312, 2013.

7. G. Forney et al., IEEE J. Sel. Ar. Comm., 2, 632-647., 1984.

11. T. Richardson and R. Urbanke, "Modern Coding Theory," Cambridge University Press, 2008.

12. C. Behrens et al., Proc. ECOC 2011 Mo.2.B.2. 